\title{
Dolor torácico anginoso como manifestación inicial de anisakiasis gástrica
}

\author{
J. M. GARCÍA GARCÍA, Mª J. ROMERO ARAUZO' \\ Unidades de Cardiología y ${ }^{\prime}$ Aparato Digestivo. Hospital El Bierzo. Ponferrada. León
}

ANGINALIKE CHEST PAIN DUE TO GASTRIC ANISAKIASIS

\begin{abstract}
RESUMEN
El dolor torácico es una de las patologías que con mayor frecuencia se atienden en los servicios de urgencia hospitalarios. Aún siendo el síndrome coronario agudo una de las primeras causas a descartar por sus importantes repercusiones, no debemos descuidar otras etiologías. Presentamos un caso de un dolor torácico de características anginosas secundario a anisakiasis gástrica. La larva del anisakis en la mucosa gástrica fue encontrada y extraída endoscópicamente. La anisakiasis gástrica debería ser incluido en el diagnóstico diferencial de un dolor torácico.
\end{abstract}

PALABRAS CLAVE: Anisakis. Anisakiasis gástrica. Dolor torácico. Dolor torácico anginoso.

\begin{abstract}
The chest pain is one of the most common reasons for consultation of the patients seen in the emergency services of hospitals. Still being the acute coronary syndrome one of the first causes to confirmed by its important repercussions, we do not have to forget other reasons. We report a case of acute anginalike chest pain due to gastric anisakiasis. The larvae of Anisakis in the gastric mucosa were found and extracted endoscopically. Gastric anisakiasis should be included in the differential diagnosis of acute chest pain.
\end{abstract}

KEY WORDS: Anisakis. Gastric anisakiasis. Chest pain. Anginalike chest pain.

García García JM, Romero Arauzo MJ. Dolor torácico anginoso como manifestación inicial de anisakiasis gástrica. An Med Interna (Madrid) 2004; 21: 185-186.

\section{INTRODUCCIÓN}

El dolor torácico es una de las patologías que con mayor frecuencia se atienden en los servicios de urgencia hospitalarios (1) y aún siendo la etiología isquémica una de las primeras causas a descartar por sus importantes repercusiones socio-sanitarias, no debemos descuidar otras etiologías. La anisakiasis es una enfermedad parasitaria del tracto digestivo producida por la ingestión de larvas de anisakis vivas. Puede afectar a cualquier parte del tubo digestivo y se han descrito múltiples manifestaciones clínicas como obstrucción intestinal, gastritis, cuadros de urticaria... Sin embargo se han documentado pocos casos de dolor torácico de características anginosas. A nivel mundial sólo se han documentado 3 casos, dos en Japón y uno en Alemania (2-4).

\section{CASO APORTADO}

Presentamos el caso de una mujer de 75 años con antecedentes de hipercolesterolemia e hipertensión arterial que acude al servicio de urgencias de nuestro hospital por presentar dolor retroesternal, opresivo que irradia a espalda de unos 30 minutos de duración, sin clínica vegetativa acompañante. En la exploración física no se evidenciaron alteraciones de interés. En las pruebas complementarias, el ECG evidenciaba un ritmo sinusal a 73 l.p.m. sin alteraciones significativas de la repolarización. La radiografía de tórax fue normal. En el análisis de sangre no se evidenciaron alteraciones en el hemograma ni en el estudio de coagulación. En la bioquímica general se evidenció un colesterol total de $216 \mathrm{mg} / \mathrm{dl}$, colesterol HDL de $53 \mathrm{mg} / \mathrm{dl}$ y un colesterol LDL de $145 \mathrm{mg} / \mathrm{dl}$. La seriación de marcadores de daño miocárdico (CK, CK-MB y Troponina I) no se elevó a rangos patológicos. En el ecocardiograma no se apreciaron alteraciones significativas. La prueba de esfuerzo fue clínica y ECG negativa para isquemia miocárdica.

Durante su estancia en planta de hospitalización de cardiología la paciente siguió presentando clínica de dolor torácico opresivo aunque de menor duración y en región retroesternal baja-epigástrica, sin evidenciarse alteraciones en el ECG durante los episodios de dolor. Valorada por la unidad de digestivo refirió que unas 6 horas antes del ingreso había ingerido pescado blanco. En la endoscopia alta se detectó en cuerpo gástrico la presencia de múltiples lesiones de pérdida de sustancia de unos $0,5 \mathrm{cms}$ de diámetro en cuyo fondo estaban incrustados parásitos cilíndricos blancos (Fig. 1). Los parásitos fue-

Trabajo aceptado: 22 de octubre de 2003

Correspondencia: Juan M. García García. Avda. La Libertad 1, Chalet B-18. 24400 Ponferrada. León. e-mail: juangarciagarcia@wanadoo.es 


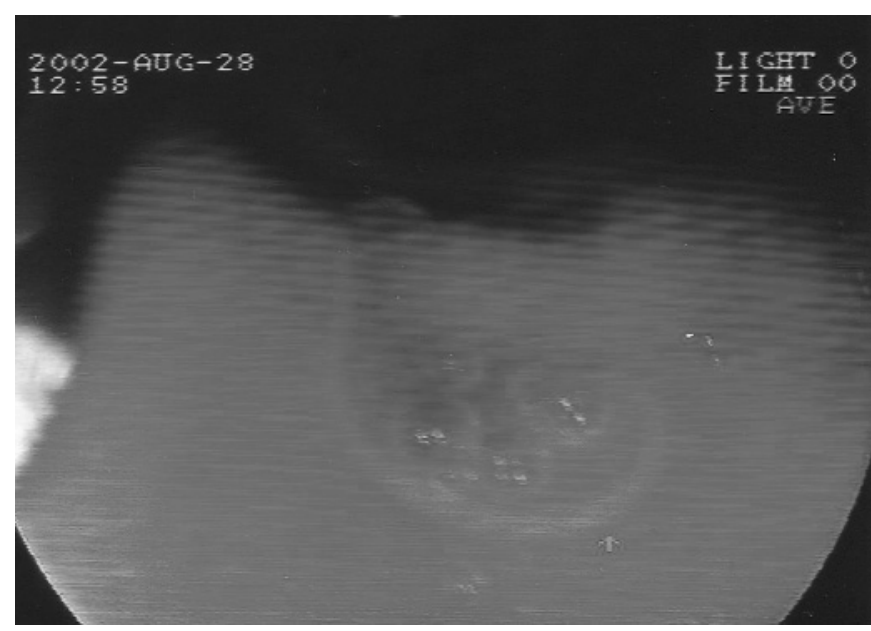

Fig. 1. Imagen de gastroscopia mostrando larva de anisakis sobre úlcera gástrica.

ron extraídos y enviados al laboratorio de microbiología siendo identificados como larvas de anisakis simple. El dolor torácico desapareció una vez extraídas las larvas.

\section{DISCUSIÓN}

La anisakiasis es una enfermedad parasitaria, infrecuente en España, que suele ser secundaria a la ingestión de pescado fresco contaminado con la larva del parásito Anisakis. La enfermedad puede tener diferentes formas clínicas de presentación, siendo la más frecuente en forma de dolor abdominal asociado a náuseas y vómitos (5). La clínica suele ocurrir varias horas después de ingerir el pescado contaminado. Sin embargo en nuestro caso la paciente presentó un dolor retroes- ternal opresivo de unos 30 minutos de duración que irradiaba a la espalda sin clínica vegetativa acompañante. La paciente fue valorada por un médico del Servicio de Urgencias e ingresada en planta de cardiología con la sospecha de un dolor torácico isquémico. El hecho de tener varios factores de riesgo cardiovascular como hipertensión arterial y dislipemia, probablemente influyó en tomar esta decisión. La sospecha de enfermedad isquémica no se pudo confirmar con ninguna de las pruebas complementarias realizadas, incluidas ECG durante los episodios de dolor torácico. Tampoco se apreciaron alteraciones en la analítica sanguínea con determinación de marcadores de necrosis miocárdica (CPK total, CPK MB y Troponina I), en el ecocardiograma ni en la ergometría. Durante su estancia en planta la paciente refirió molestias en región epigástrica por lo que solicitamos la valoración de un especialista de aparato digestivo. Se realizó una endoscopia digestiva alta y evidenció múltiples larvas parasitarias en la mucosa del estómago. El dolor torácico no cardiogénico es un problema frecuente en los servicios de urgencia hospitalarios y con frecuencia son secundarios a patología digestiva como el reflujo gastroesofágico y alteraciones en la motilidad esofágica (6). Teniendo en cuenta este caso clínico, en el diagnóstico diferencial del dolor torácico, también debemos pensar en ulceraciones de la mucosa gástrica por anisakis. En casos similares consideramos que es fundamental realizar una buena historia clínica pues aunque el dolor torácico isquémico es la primera causa a descartar no debemos descuidar otras etiologías como la patología digestiva. En estos casos la endoscopia alta nos da el diagnóstico de certeza (7).

Por lo tanto, aunque la anisakiasis gástrica es una enfermedad rara, debe ser tenida en cuenta en dolores torácicos inespecíficos, con ECG sin alteraciones significativas y ausencia de elevación de marcadores de necrosis miocárdica; sobre todo si el paciente ha consumido pescado fresco recientemente.

\section{Bibliografía}

1. Bayón Fernández J, Alegría Ezquerra E, Bosch Genover X, et al. Unidades de dolor torácico. Organización y protocolo para el diagnóstico de los síndromes coronarios agudos. Rev Esp Cardiol 2002; 55: 143 154.

2. Sugano S, Suzuki T, Kagesawa M, Kawafune T, Ohshima Y. Noncardiac chest pain due to acute gastric anisakiasis. Dig Dis Sci. 1993; 38 (7): 1354-1356.

3. Machi T, Okino S, Saito Y, Horita Y, Taguchi T, Nakazawa T, Nakamura Y, Hirai H, Miyamori H, Kitagawa S. Severe chest pain due to gastric anisakiasis. Intern Med 1997; 36 (1): 28-30.

4. Plath F, Holle A, Zendeh D, Moller FW, Barten M, Reisinger EC, Liebe

S. Anisakiasis of the stomach. A case report from Germany. Z Gastroenterol 2001; 39 (2): 177-180.

5. Acebes Rey JM, Fernández Orcajo P, Díaz González G, et al. Dos casos de anisakiasis en el Hospital del Río Hortega. Rev Esp Enferm Dig 1996; 88: 59-60.

6. Lam HGT, Dekker W, Kaan G, et al. Acute non cardiac chest pain in a coronari care unit. Evaluation by 24 hour presssure and $\mathrm{pH}$ recording of the esopahgus. Gastroenterology 1992; 102: 453-460.

7. Domínguez Ortega J, Cimarra M, Sevilla MC, et al. Anisakis simplex: una causa de pseudobstrucción intestinal. Rev Esp Enferm Dig 2000; 92: 132-135. 